\title{
The Adjusted Log-logistic Generalized Exponential Distribution with Application to Lifetime Data
}

\author{
I. E. Okorie ${ }^{1}$, A. C. Akpanta ${ }^{2}$, J. Ohakwe ${ }^{3}$, D. C. Chikezie ${ }^{2}$, C. U. Onyemachi ${ }^{2}$, E. O. Obi ${ }^{4}$ \\ ${ }^{1}$ School of Mathematics, University of Manchester, Manchester, M13 9PL, UK \\ ${ }^{2}$ Department of Statistics, Abia State University, Uturu, Abia State, Nigeria \\ ${ }^{3}$ Department of Mathematics \& Statistics, Faculty of Sciences, Federal University Otuoke, Bayelsa State, P. M. 126 \\ Yenagoa, Bayelsa, Nigeria \\ ${ }^{4}$ Department of Strategic Knowledge Management, NACA, Abuja, Nigeria \\ Correspondence: I. E. Okorie, School of Mathematics, University of Manchester, Manchester, M13 9PL, UK. E-mail: \\ idika.okorie@manchester.ac.uk
}

Received: March 14, 2017 Accepted: May 18, 2017 Online Published: June 7, 2017

doi:10.5539/ijsp.v6n4p1 URL: https://doi.org/10.5539/ijsp.v6n4p1

\begin{abstract}
This paper introduces a new generator of probability distribution-the adjusted log-logistic generalized (ALLoG) distribution and a new extension of the standard one parameter exponential distribution called the adjusted log-logistic generalized exponential (ALLoGExp) distribution. The ALLoGExp distribution is a special case of the ALLoG distribution and we have provided some of its statistical and reliability properties. Notably, the failure rate could be monotonically decreasing, increasing or upside-down bathtub shaped depending on the value of the parameters $\delta$ and $\theta$. The method of maximum likelihood estimation was proposed to estimate the model parameters. The importance and flexibility of the ALLoGExp distribution was demonstrated with a real and uncensored lifetime data set and its fit was compared with five other exponential related distributions. The results obtained from the model fittings shows that the ALLoGExp distribution provides a reasonably better fit than the one based on the other fitted distributions. The ALLoGExp distribution is therefore recommended for effective modelling of lifetime data sets.
\end{abstract}

Keywords: asymptotic adjustment, exponential distribution, log-logistic distribution, reliability, failure rate

\section{Introduction}

Due to the lack of fits that characterize the standard probability distributions in modelling various complex real data sets a lot of effort have been expended by researchers in developing new distributions as a way of circumventing the problem of inadequate fits of the already existing distributions. The new distributions often referred to as as the generalized class of distributions have consistently been shown to provide better fits than the existing (standard) ones. Almost all the available methods of generating new distributions in statistical literature depends on the cumulative distribution function of the standard distributions; for a holistic up-to-date review of these methods see; Nadarajah and Rocha (2016).

The main motivation of this paper stem from the trending literature of probability distribution construction and generalization; which in principle, entails the injection of one distribution into another distribution, in order to extend the injected distribution to a wider family of distribution with added flexibility. In the literature, the generalized distributions are often referred to as the G-distributions. For example,

1. Eugene et al. (2002) defined a new class of distribution called the Beta-G family of distributions as the logit of the beta random variable with cumulative density function $(c d f)$ as

$$
F(x)=\frac{\Gamma(\alpha+\beta)}{\Gamma(\alpha) \Gamma(\beta)} \int_{0}^{G(x)} y^{\alpha-1}(1-y)^{\beta-1} d y ; 0<\alpha, \beta<\infty,
$$

and probability density function $(p d f)$ as

$$
f(x)=\frac{\Gamma(\alpha+\beta)}{\Gamma(\alpha) \Gamma(\beta)} G^{\alpha-1}(x)[1-G(x)]^{\beta-1} \frac{d G(x)}{d x} ; 0<\alpha, \beta<\infty .
$$

2. Cordeiro and de Castro (2011) introduced the Kumaraswamy-G family of distributions with $c d f$ 


$$
F(x)=a b \int_{0}^{G(x)} y^{a-1}\left(1-y^{a}\right)^{b-1} d y ; x \in(0,1), a, b>0,
$$

and $p d f$

$$
f(x)=\operatorname{abg}(x) G^{a-1}(x)\left[1-G^{a}(x)\right]^{b-1} ; x \in(0,1), a, b>0 .
$$

3. Zografos and Balakrishnan (2009) proposed the Gamma-G family of distributions based on baseline continuous distribution with reliability function $\bar{G}(x)=1-G(x)$. The $c d f$ of the Gamma-G distributions is defined as

$$
F(x)=\frac{1}{\Gamma(\delta)} \int_{0}^{-\log \bar{G}(x)} y^{\delta-1} e^{-y} d y ; x \in \mathbf{R}, \delta>0,
$$

and $p d f$ as

$$
f(x)=\frac{1}{\Gamma(\delta)}[-\log \bar{G}(x)]^{\delta-1} g(x) ; x \in \mathbf{R}, \delta>0 .
$$

4. An alternative version of Zografos and Balakrishnan (2009) was proposed by Ristic and Balakrishnan (2012). The $c d f$ of the gamma generator due to Ristić and Balakrishnan (2012) is defined as

$$
F(x)=1-\frac{1}{\Gamma(\delta)} \int_{0}^{-\log G(x)} y^{\delta-1} e^{-y} d y ; x \in \mathbf{R}, \delta>0,
$$

while its $p d f$ is given by;

$$
f(x)=\frac{1}{\Gamma(\delta)}[-\log G(x)]^{\delta-1} g(x) ; x \in \mathbf{R}, \delta>0 .
$$

5. Alzaatreh et al. (2013) introduced the Weibull-G distributions whose $c d f$ is defined as

$$
F(x)=\frac{\alpha}{\beta^{\alpha}} \int_{0}^{-\log \bar{G}(x)} y^{\alpha-1} e^{-\left(\frac{y}{\beta}\right)^{\alpha}} d y ; x \in \mathbf{R}, \alpha, \beta>0,
$$

and $p d f$ as

$$
f(x)=\frac{\alpha}{\beta^{\alpha}} \frac{g(x)}{\bar{G}(x)}\left[\frac{-\log \bar{G}(x)}{\beta}\right]^{\alpha-1} e^{-\left[\frac{-\log \bar{G}(x)}{\beta}\right]^{\alpha}} ; x \in \mathbf{R}, \alpha, \beta>0 .
$$

In all cases, $G(x)$ is the $c d f$ of the injected distribution (or baseline distribution) with $g(x)$ as the corresponding $p d f$, $F(x)$ is the $c d f$ of the new generalized version of $G(x)$ with $f(x)$ as the corresponding $p d f$, and $f(x)$ have the same support as $g(x)$.

and so on.

The aim of this paper is two-fold; first to introduce a new generator of distributions-the adjusted log-logistic generalized $(A L L o G)$ distribution which as far as we know have not appeared in the literature before now and secondly, to introduce and give explicit statistical properties of the adjusted log-logistic generalized exponential (ALLoGExp) distribution as a sub-model of the $A L L o G$ distribution which generalizes the standard one parameter exponential distribution. The $c d f G(x)$ of the log-logistic distribution is given by;

$$
G(x)=\frac{x^{\theta}}{\delta^{\theta}+x^{\theta}} ; x>0, \delta, \theta>0
$$

with the corresponding $p d f g(x)$ defined as

$$
g(x)=\frac{\frac{\theta}{\delta}\left(\frac{x}{\delta}\right)^{\theta-1}}{\left[1+\left(\frac{x}{\delta}\right)^{\theta}\right]^{2}} ; x>0, \delta, \theta>0,
$$

where $\delta$ is the scale parameter and $\theta$ is the shape parameter. 
We define the $c d f F(x)$ and $p d f f(x)$ of the new generator $A L L o G$ with (1) and (2) as

$$
F(x)=\frac{\theta\left(\delta^{\theta}+1\right)}{\delta^{\theta}} \int_{0}^{G(x)} y^{\theta-1}\left[1+\left(\frac{y}{\delta}\right)^{\theta}\right]^{-2} d y ; x>0, \delta, \theta>0 .
$$

and

$$
f(x)=\left(\delta^{\theta}+1\right) \delta^{\theta} \theta g(x) G^{\theta-1}(x)\left[\delta^{\theta}+G^{\theta}(x)\right]^{-2} ; x>0, \delta, \theta>0,
$$

respectively. Where, $\delta^{\theta}+1$ in (3) is an asymptotic adjustment (normalizing constant); introduced, to ensure that $\lim _{x \rightarrow \infty} F(x)=1$.

The quantile function is given by;

$$
F^{-1}(p)=G^{-1}\left[\left(\frac{p \delta^{\theta}}{\delta^{\theta}+1-p}\right)^{\frac{1}{\theta}}\right] ; p \in(0,1], \delta, \theta>0,
$$

Unlike the $c d f$ and $p d f$ of the Beta-G and Gamma-G family of distributions, the $c d f$ and $p d f$ of the new generator $A L L o G$ is easy to work with analytically because, it does not contain any special function.

The remainder of this paper is organized as follows: In Section 2 we present the closed form expression for the probability density function $p d f$ and the cumulative density function $c d f$ and other statistical properties of the new probability distribution (ALLoGExp). In Section 3 the parameters of the ALLoGExp distribution is estimated through the method of maximum likelihood estimation. Section 4 contains some reliability characteristics and possible shapes of the ALLoGExp distribution. In Section 5 we illustrate the applicability of the ALLoGExp distribution with a real data set. Section 6 is the Monte-Carlo simulation study of the parameters and Finally, Section 7 gives the concluding remarks.

\section{The $A L L O G E x p$ and its Properties}

The standard one parameter exponential (Exp) distribution has its $c d f G(x)$ as $G(x)=1-e^{-\eta x} ; x \geq 0, \eta>0$ and $p d f$ $g(x)$ as $g(x)=\eta e^{-\eta x} ; x \geq 0, \eta>0$; where, $\eta$ is the rate parameter. Thus, it follows that the $c d f F(x)$ of the ALLoGExp distribution is given by;

$$
F(x)=\frac{\left(\delta^{\theta}+1\right)\left(1-e^{-\eta x}\right)^{\theta}}{\delta^{\theta}+\left(1-e^{-\eta x}\right)^{\theta}} ; x \geq 0, \delta, \theta, \eta>0,
$$

with the corresponding $p d f f(x)$ as

$$
f(x)=\left(\delta^{\theta}+1\right) \delta^{\theta} \theta \eta e^{-\eta x}\left(1-e^{-\eta x}\right)^{\theta-1}\left[\delta^{\theta}+\left(1-e^{-\eta x}\right)^{\theta}\right]^{-2} ; x \geq 0, \delta, \theta, \eta>0,
$$

where $\delta$ is the scale parameter, $\theta$ is the shape parameters and $\eta$ is the rate parameter.

\subsection{Quantile Function and Random Number Generation}

By using (6), we obtain the quantile function of the ALLoGExp distribution as

$$
F^{-1}(p)=-\frac{1}{\eta} \log \left(1-\left[\frac{p \delta^{\theta}}{\delta^{\theta}+1-p}\right]^{\frac{1}{\theta}}\right) ; p \in(0,1], \delta, \theta, \eta>0 .
$$

Random samples from the ALLoGExp distribution can be obtained through the inverse transformation method of random number generation by simply substituting $p$ in $(8)$ with a Uniform $(0,1)$ variate. Also, it is easy to obtain the median of the ALLoGExp distribution by simply substituting $p=0.5$ in (8), which is given by;

$$
F^{-1}(0.5)=-\frac{1}{\eta} \log \left(1-\left[\frac{\delta^{\theta}}{2 \delta^{\theta}+1}\right]^{\frac{1}{\theta}}\right) ; \delta, \theta, \eta>0 .
$$

\subsection{Moments}

In statistics and applications the moments of a random variable say $X$ are important, they are used to characterize the underlying distribution. For example, to measure the center, spread/variation of the distribution, and to ascertain the degree of deviation from normality (skewness and kurtosis) of the distribution, etc.; 

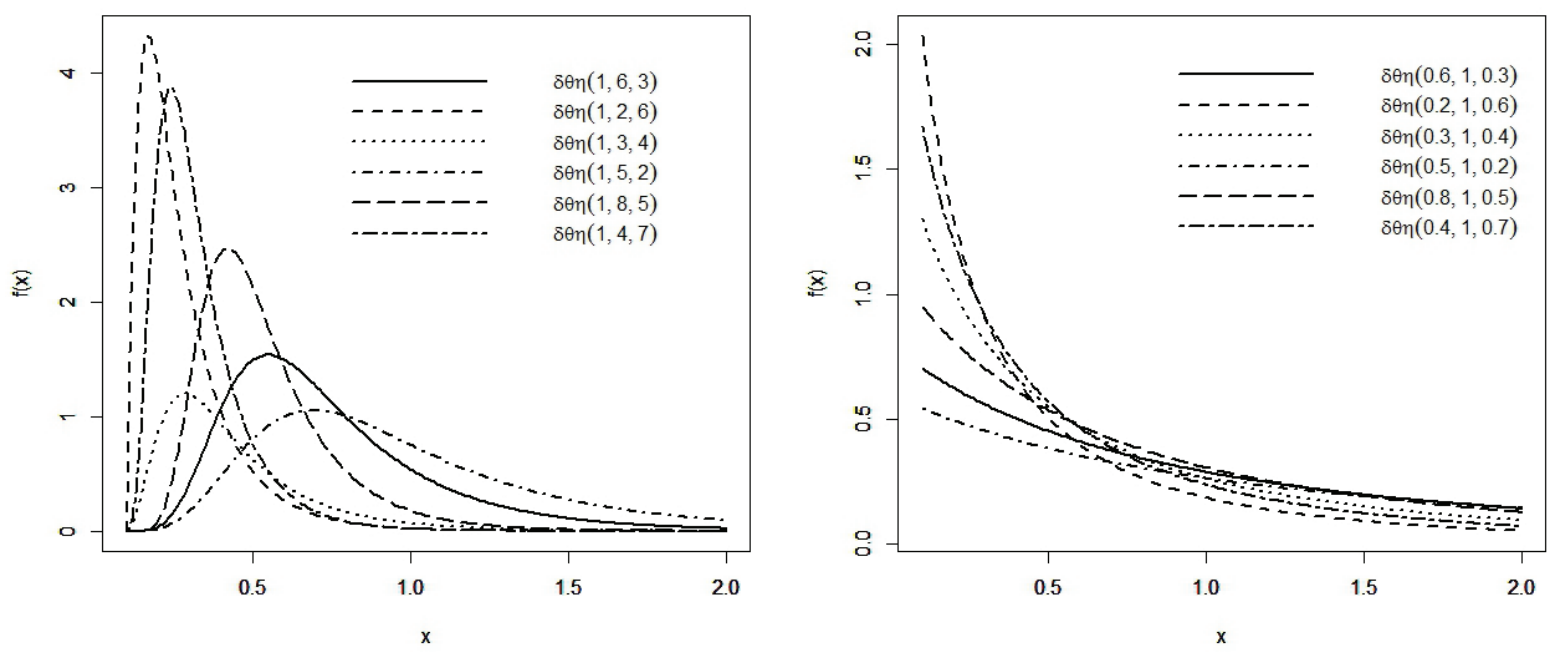

Figure 1. Possible shapes of the probability density function $p d f f(x)$ for some parameter values.
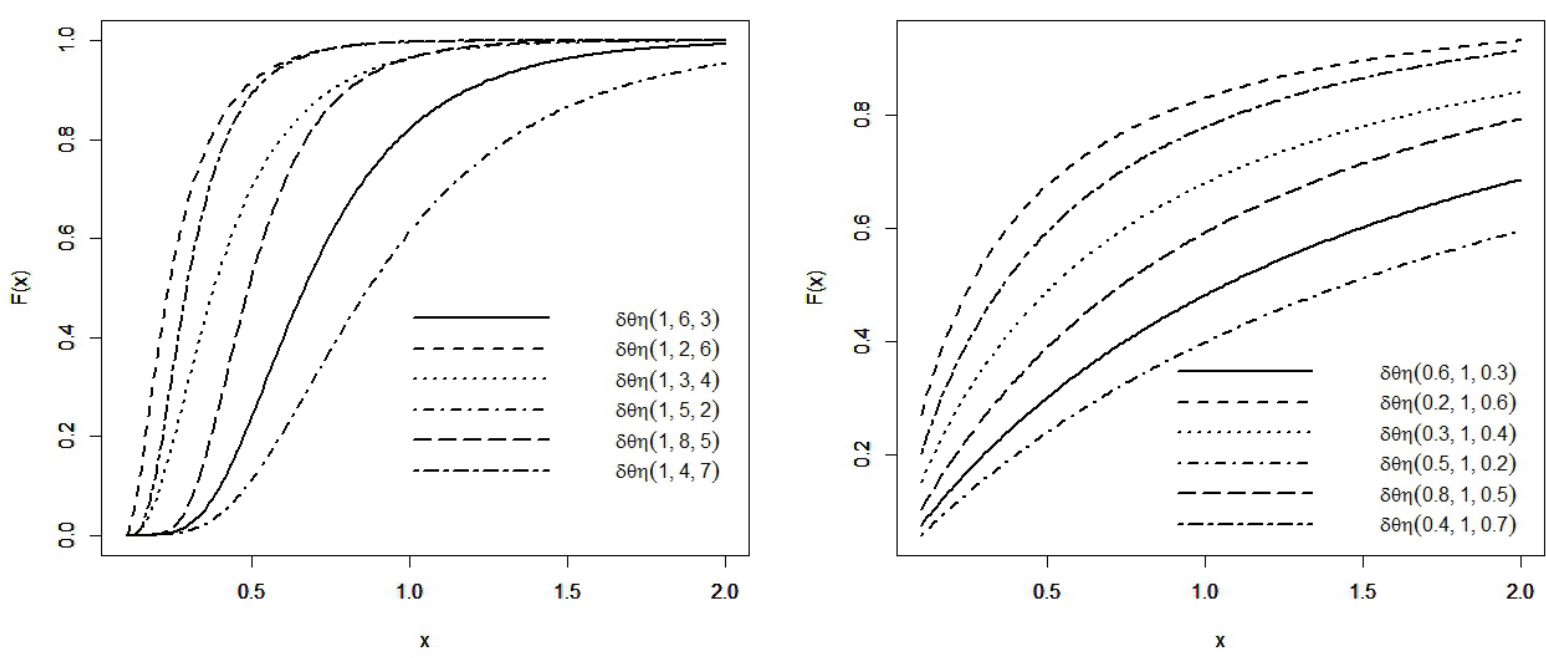

Figure 2. Possible shapes of the cumulative density function $c d f F(x)$ for some parameter values.

Theorem 2.1. If $X$ follows the ALLoGExp distribution with pdf defined in (7) then its kth crude moment is given by;

$$
\mu_{k}^{\prime}=\left.\frac{\left(\delta^{\theta}+1\right) \theta}{(-\eta)^{k} \delta^{\theta}} \sum_{m=0}^{\infty}\left(\frac{-1}{\delta^{\theta}}\right)^{m}(m+1) \frac{\partial^{k}}{\partial \ell^{k}} \mathbf{B}(\ell+1, \theta(m+1))\right|_{\ell=0},
$$

where $\mathbf{B}(\cdot)$ is the beta function.

Proof. By using (7) we have

$$
\mu_{k}^{\prime}=\int_{\mathbf{R}} x^{k} f(x) d x .
$$


Where (10) is computed as follows

$$
\begin{aligned}
\mu_{k}^{\prime} & =\int_{0}^{\infty} x^{k}\left(\delta^{\theta}+1\right) \delta^{\theta} \theta \eta e^{-\eta x}\left(1-e^{-\eta x}\right)^{\theta-1}\left[\delta^{\theta}+\left(1-e^{-\eta x}\right)^{\theta}\right]^{-2} d x \\
& =\left(\delta^{\theta}+1\right) \delta^{\theta} \theta \eta \int_{0}^{\infty} x^{k} e^{-\eta x}\left(1-e^{-\eta x}\right)^{\theta-1}\left[\delta^{\theta}+\left(1-e^{-\eta x}\right)^{\theta}\right]^{-2} d x
\end{aligned}
$$

substituting $y=e^{-\eta x}$ in (11) we have

$$
\begin{aligned}
\mu_{k}^{\prime} & =\frac{\left(\delta^{\theta}+1\right) \delta^{\theta} \theta}{(-\eta)^{k}} \int_{0}^{1}(\log (y))^{k}(1-y)^{\theta-1}\left[\delta^{\theta}+(1-y)^{\theta}\right]^{-2} d y \\
& =\left.\frac{\left(\delta^{\theta}+1\right) \delta^{\theta} \theta}{(-\eta)^{k}} \int_{0}^{1} \frac{\partial^{k}}{\partial \ell^{k}} y^{\ell}(1-y)^{\theta-1}\left[\delta^{\theta}+(1-y)^{\theta}\right]^{-2} d y\right|_{\ell=0},
\end{aligned}
$$

by expanding (12) we have

$$
\begin{aligned}
\mu_{k}^{\prime} & =\left.\frac{\left(\delta^{\theta}+1\right) \delta^{\theta} \theta}{(-\eta)^{k}} \frac{\partial^{k}}{\partial \ell^{k}} \int_{0}^{1} y^{\ell}(1-y)^{\theta-1} \sum_{m=0}^{\infty}(-1)^{m}\left(\begin{array}{c}
2+m-1 \\
m
\end{array}\right)(1-y)^{\theta m} \delta^{-\theta(2+m)} d y\right|_{\ell=0} \\
& =\left.\frac{\left(\delta^{\theta}+1\right) \theta}{(-\eta)^{k} \delta^{\theta}} \sum_{m=0}^{\infty}\left(\frac{-1}{\delta^{\theta}}\right)^{m}(m+1) \frac{\partial^{k}}{\partial \ell^{k}} \int_{0}^{1} y^{\ell}(1-y)^{\theta(m+1)-1} d y\right|_{\ell=0} \\
& =\left.\frac{\left(\delta^{\theta}+1\right) \theta}{(-\eta)^{k} \delta^{\theta}} \sum_{m=0}^{\infty}\left(\frac{-1}{\delta^{\theta}}\right)^{m}(m+1) \frac{\partial^{k}}{\partial \ell^{k}} \mathbf{B}(\ell+1, \theta(m+1))\right|_{\ell=0} .
\end{aligned}
$$

Corollary 2.1.1. Evaluating (13) at $\mathrm{k}=1,2,3$, and 4 we have the first four crude moments of the ALLoGExp distribution as follows:

$$
\begin{aligned}
\mu_{1}^{\prime}= & \frac{\left(\delta^{\theta}+1\right) \theta}{-\eta \delta^{\theta}} \sum_{m=0}^{\infty}\left(\frac{-1}{\delta^{\theta}}\right)^{m}(m+1)(\Psi(1)-\Psi(1+\theta(m+1))) \mathbf{B}(1, \theta(m+1)), \\
\mu_{2}^{\prime}= & \frac{\left(\delta^{\theta}+1\right) \theta}{(-\eta)^{2} \delta^{\theta}} \sum_{m=0}^{\infty}\left(\frac{-1}{\delta^{\theta}}\right)^{m}(m+1)(\Psi(1,1)-\Psi(1,1+\theta(m+1))) \mathbf{B}(1, \theta(m+1)) \\
& +(\Psi(1)-\Psi(1+\theta(m+1)))^{2} \mathbf{B}(1, \theta(m+1)), \\
\mu_{3}^{\prime} & =\frac{\left(\delta^{\theta}+1\right) \theta}{(-\eta)^{3} \delta^{\theta}} \sum_{m=0}^{\infty}\left(\frac{-1}{\delta^{\theta}}\right)^{m}(m+1)(\Psi(2,1)-\Psi(2,1+\theta(m+1))) \mathbf{B}(1, \theta(m+1)) \\
& +3(\Psi(1,1)-\Psi(1,1+\theta(m+1)))(\Psi(1)-\Psi(1+\theta(m+1))) \mathbf{B}(1, \theta(m+1)) \\
& +(\Psi(1)-\Psi(1+\theta(m+1)))^{3} \mathbf{B}(1, \theta(m+1)),
\end{aligned}
$$

and

$$
\begin{aligned}
\mu_{4}^{\prime} & =\frac{\left(\delta^{\theta}+1\right) \theta}{(-\eta)^{4} \delta^{\theta}} \sum_{m=0}^{\infty}\left(\frac{-1}{\delta^{\theta}}\right)^{m}(m+1)(\Psi(3,1)-\Psi(3,1+\theta(m+1))) \mathbf{B}(1, \theta(m+1)) \\
& +4(\Psi(2,1)-\Psi(2,1+\theta(m+1)))(\Psi(1)-\Psi(1+\theta(m+1))) \mathbf{B}(1, \theta(m+1)) \\
& +3(\Psi(1,1)-\Psi(1,1+\theta(m+1)))^{2} \mathbf{B}(1, \theta(m+1))+6(\Psi(1,1)) \\
& -\Psi(1,1+\theta(m+1)))(\Psi(1)-\Psi(1+\theta(m+1)))^{2} \mathbf{B}(1, \theta(m+1)) \\
& +(\Psi(1)-\Psi(1+\theta(m+1)))^{4} \mathbf{B}(1, \theta(m+1))
\end{aligned}
$$

where $\mathbf{B}(\cdot)$ is the beta function and $\Psi(\cdot)$ is the psi or digamma function. 
Corollary 2.1.2. By appropriately using (14)-(17) we can obtain the variance $\left(\sigma^{2}\right)$, coefficient of variation (CV), skewness $\left(\gamma_{1}\right)$ and kurtosis $\left(\gamma_{2}\right)$ of the ALLoGExp distribution as

$$
\begin{aligned}
\sigma^{2} & =\mu_{2}^{\prime}-\mu_{1}^{\prime 2}, \\
C V & =\sqrt{\frac{\mu_{2}^{\prime}}{\mu_{1}^{\prime 2}}-1} \\
\gamma_{1} & =\frac{\mu_{3}^{\prime}-3 \mu_{2}^{\prime} \mu_{1}^{\prime}+2 \mu_{1}^{\prime 3}}{\left(\mu_{2}^{\prime}-\mu_{1}^{\prime 2}\right)^{\frac{3}{2}}}
\end{aligned}
$$

and

$$
\gamma_{2}=\frac{\mu_{4}^{\prime}-4 \mu_{3}^{\prime} \mu_{1}^{\prime}+6 \mu_{2}^{\prime} \mu_{1}^{\prime 2}-3 \mu_{1}^{\prime 4}}{\left(\mu_{2}^{\prime}-\mu_{1}^{\prime 2}\right)^{2}}
$$

respectively.

Theorem 2.2. If $X$ follows the ALLoGExp distribution with pdf defined in (7) then its moment generating function (mgf) is given by;

$$
M_{X}(t)=\left.\theta\left(\delta^{\theta}+1\right) \sum_{k, m=0}^{\infty} \frac{(-1)^{m}(m+1) t^{k}}{(-\eta)^{k} \delta^{\theta(m+1)} k !} \frac{\partial^{k}}{\partial \ell^{k}} \mathbf{B}(\ell+1, \theta(m+1))\right|_{\ell=0} .
$$

Proof. Using the definition of the $m g f$ of the continuous random variable say $X$ which is defined as

$$
M_{X}(t)=E\left(e^{t x}\right)=E\left(\sum_{k=0}^{\infty} \frac{(t x)^{k}}{k !}\right)=\sum_{k=0}^{\infty} \frac{t^{k}}{k !} \mu_{k}^{\prime},
$$

and by substituting (13) into (19) it is clear that the $m g f$ of the ALLoGExp distribution is as presented in (18).

\subsection{Entropy Measure}

In this section we present the Rényi entropy measure of the ALLoGExp distribution. The Rényi entropy measure is used to quantify the uncertainty of variation in a random variable say $X$ and the Rényi entropy measure of a continuous random variable is generally given by;

$$
I_{R}(\varphi)=\frac{1}{1-\varphi} \log \left(\int_{\mathbf{R}} f^{\varphi}(x) d x\right) ; \varphi>0 \backslash\{1\} .
$$

Theorem 2.3. If $X$ follows the ALLoGExp distribution with pdf defined in (7) then its Rényi entropy measure is given by;

$$
\begin{aligned}
I_{R}(\varphi) & =\frac{1}{1-\varphi} \log \left[\frac{\left(\left[\delta^{\theta}+1\right] \delta^{\theta} \theta\right)^{\varphi}}{\eta^{-\varphi+1}} \sum_{i, j=0}^{\infty}(-1)^{i+j}\right. \\
& \left.\times \frac{\delta^{-\theta(2 \varphi+j)} \Gamma(\varphi(\theta-1)+1) \Gamma(2 \varphi+j) \mathbf{B}(\varphi+i+1, \theta j+1)}{\Gamma(i+1) \Gamma(j+1) \Gamma(\varphi(\theta-1)-i+1) \Gamma(2 \varphi)}\right],
\end{aligned}
$$

where $\Gamma(\cdot)$ is the gamma function and $\mathbf{B}(\cdot)$ is the beta function.

Proof. Substituting (7) into (20), setting $\int_{\mathbf{R}} f^{\varphi}(x) d x$ to $\mathcal{A}_{\varphi}$ and evaluating the integral on the support $[0, \infty)$ gives

$$
\begin{aligned}
\mathcal{A}_{\varphi} & =\int_{0}^{\infty} f^{\varphi}(x) d x \\
& =\int_{0}^{\infty}\left(\left[\delta^{\theta}+1\right] \delta^{\theta} \theta \eta\right)^{\varphi} e^{-\varphi \eta x}\left(1-e^{-\eta x}\right)^{\varphi(\theta-1)}\left[\delta^{\theta}+\left(1-e^{-\eta x}\right)^{\theta}\right]^{-2 \varphi} d x \\
& =\left(\left[\delta^{\theta}+1\right] \delta^{\theta} \theta \eta\right)^{\varphi} \int_{0}^{\infty} e^{-\varphi \eta x}\left(1-e^{-\eta x}\right)^{\varphi(\theta-1)}\left[\delta^{\theta}+\left(1-e^{-\eta x}\right)^{\theta}\right]^{-2 \varphi} d x .
\end{aligned}
$$


By expanding (21) we have

$$
\begin{aligned}
\mathcal{A}_{\varphi} & =\left(\left[\delta^{\theta}+1\right] \delta^{\theta} \theta \eta\right)^{\varphi} \int_{0}^{\infty} e^{-\varphi \eta x} \sum_{i=0}^{\infty}\left(\begin{array}{c}
\varphi(\theta-1) \\
i
\end{array}\right)(-1)^{i} e^{-\eta i x} \\
& \times \sum_{j=0}^{\infty}\left(\begin{array}{c}
2 \varphi+j-1 \\
j
\end{array}\right)(-1)^{j}\left(1-e^{-\eta x}\right)^{\theta j} \delta^{-\theta(2 \varphi+j)} d x \\
\mathcal{A}_{\varphi} & =\left(\left[\delta^{\theta}+1\right] \delta^{\theta} \theta \eta\right)^{\varphi} \sum_{i, j=0}^{\infty}(-1)^{i+j}\left(\begin{array}{c}
\varphi(\theta-1) \\
i
\end{array}\right)\left(\begin{array}{c}
2 \varphi+j-1 \\
j
\end{array}\right) \delta^{-\theta(2 \varphi+j)} \\
& \times \int_{0}^{\infty} e^{-\eta(\varphi+i) x}\left(1-e^{-\eta x}\right)^{\theta j} d x
\end{aligned}
$$

and by substituting $y=e^{-\eta x}$ into (22) we have

$$
\begin{aligned}
\mathcal{A}_{\varphi} & =\frac{\left(\left[\delta^{\theta}+1\right] \delta^{\theta} \theta\right)^{\varphi}}{\eta^{-\varphi+1}} \sum_{i, j=0}^{\infty}(-1)^{i+j}\left(\begin{array}{c}
\varphi(\theta-1) \\
i
\end{array}\right)\left(\begin{array}{c}
2 \varphi+j-1 \\
j
\end{array}\right) \delta^{-\theta(2 \varphi+j)} \\
& \times \int_{0}^{1} y^{\varphi+i}(1-y)^{\theta j} d y
\end{aligned}
$$

which further simplifies to

$$
\begin{aligned}
\mathcal{A}_{\varphi} & =\frac{\left(\left[\delta^{\theta}+1\right] \delta^{\theta} \theta\right)^{\varphi}}{\eta^{-\varphi+1}} \sum_{i, j=0}^{\infty}(-1)^{i+j}\left(\begin{array}{c}
\varphi(\theta-1) \\
i
\end{array}\right)\left(\begin{array}{c}
2 \varphi+j-1 \\
j
\end{array}\right) \delta^{-\theta(2 \varphi+j)} \\
& \times \mathbf{B}(\varphi+i+1, \theta j+1)
\end{aligned}
$$

and finally

$$
\begin{aligned}
\mathcal{A}_{\varphi} & =\frac{\left(\left[\delta^{\theta}+1\right] \delta^{\theta} \theta\right)^{\varphi}}{\eta^{-\varphi+1}} \sum_{i, j=0}^{\infty}(-1)^{i+j} \\
& \times \frac{\delta^{-\theta(2 \varphi+j)} \Gamma(\varphi(\theta-1)+1) \Gamma(2 \varphi+j) \mathbf{B}(\varphi+i+1, \theta j+1)}{\Gamma(i+1) \Gamma(j+1) \Gamma(\varphi(\theta-1)-i+1) \Gamma(2 \varphi)} .
\end{aligned}
$$

Thus, substituting (23) into (20) completes the proof.

\subsection{Order Statistics}

Order statistics is an essential tool in reliability and life testing analysis. For instance, suppose the following $n$-sized random sample $X_{1}, X_{2}, \ldots, X_{n}$ are drawn from the ALLoGExp distribution with $c d f$ and $p d f$ corresponding to (6) and (7). Let $X_{1, n} \leq X_{2, n} \leq \ldots \leq X_{n, n}$ represent the ith order statistics denoted by $X_{i, n}$ then, $X_{i, n}$ could be interpreted as the lifetime of the $(n-i+1)$ th item of the total $n$th independent and identical components. The density of $X_{i, n}$ could be expressed as

$$
\begin{aligned}
f_{X_{(i)}}(x) & =\frac{n !}{(i-1) !(n-i) !} F^{i-1}(x)(1-F(x))^{n-i} f(x) \\
& =\frac{n !}{(i-1) !(n-i) !} \sum_{\ell=0}^{n-i}\left(\begin{array}{c}
n-i \\
\ell
\end{array}\right) F^{i+\ell-1}(x) f(x) .
\end{aligned}
$$

The $c d f$ of the ALLoGExp distribution in (6) to the $(i+\ell-1)$ th power is given by;

$$
F^{i+\ell-1}(x)=\left[\delta^{\theta}+1\right]^{i+\ell-1}\left(1-e^{-\eta x}\right)^{\theta(i+\ell-1)}\left[\delta^{\theta}+\left(1-e^{-\eta x}\right)^{\theta}\right]^{-(i+\ell-1)},
$$


and if $\theta \in \mathbf{N}$, where $\mathbf{N} \backslash\{0\}$ is a natural number; then we have the series representation of (34) as

$$
\begin{aligned}
F^{i+\ell-1}(x) & =\sum_{k=0}^{\theta(i+\ell-1)}\left(\begin{array}{c}
\theta(i+\ell-1) \\
k
\end{array}\right)(-1)^{k}\left[\delta^{\theta}+1\right]^{i+\ell-1} e^{-\eta k x} \\
& \times \sum_{m=0}^{i+\ell-1}(-1)^{m}\left(\begin{array}{c}
i+\ell+m-2 \\
m
\end{array}\right) \delta^{-\theta(i+\ell+m-1)}\left(1-e^{-\eta x}\right)^{\theta m}, \\
F^{i+\ell-1}(x)= & \sum_{k=0}^{\theta(i+\ell-1)} \sum_{m=0}^{i+\ell-1}(-1)^{k+m}\left[\delta^{\theta}+1\right]^{i+\ell-1} \delta^{-\theta(i+\ell+m-1)} e^{-\eta k x} \\
& \times\left(\begin{array}{c}
\theta(i+\ell-1) \\
k
\end{array}\right)\left(\begin{array}{c}
i+\ell+m-2 \\
m
\end{array}\right) \sum_{n=0}^{\theta m}\left(\begin{array}{c}
\theta m \\
n
\end{array}\right)(-1)^{n} e^{-\eta n x}, \\
F^{i+\ell-1}(x) & =\sum_{k=0}^{\theta(i+\ell-1)} \sum_{m=0}^{i+\ell-1}(-1)^{k+m+n}\left[\delta^{\theta}+1\right]^{i+\ell-1} \delta^{-\theta(i+\ell+m-1)} \\
& \times\left(\begin{array}{c}
\theta(i+\ell-1) \\
k
\end{array}\right)\left(\begin{array}{c}
i+\ell+m-2 \\
m
\end{array}\right)\left(\begin{array}{c}
\theta m \\
n
\end{array}\right) e^{-\eta(k+n) x} .
\end{aligned}
$$

Also, the series representation of (7) is given by;

$$
\begin{aligned}
f(x) & =\left[\delta^{\theta}+1\right] \delta^{\theta} \theta \eta e^{-\eta x} \sum_{h=0}^{\theta-1}\left(\begin{array}{c}
\theta-1 \\
h
\end{array}\right)(-1)^{h} e^{-\eta h x} \sum_{i=0}^{2}(-1)^{i}\left(\begin{array}{c}
i+1 \\
i
\end{array}\right) \delta^{-\theta(2+i)}\left(1-e^{-\eta x}\right)^{\theta i} \\
& =\left[\delta^{\theta}+1\right] \delta^{\theta} \theta \eta e^{-\eta x} \sum_{h=0}^{\theta-1} \sum_{i=0}^{2}(-1)^{h+i}\left(\begin{array}{c}
\theta-1 \\
h
\end{array}\right)\left(\begin{array}{c}
i+1 \\
i
\end{array}\right) \delta^{-\theta(2+i)} e^{-\eta h x} \sum_{j=0}^{\theta i}\left(\begin{array}{c}
\theta i \\
j
\end{array}\right)(-1)^{j} e^{-\eta j x} \\
& =\left[\delta^{\theta}+1\right] \delta^{\theta} \theta \eta \sum_{h=0}^{\theta-1} \sum_{i=0}^{2} \sum_{j=0}^{\theta i}(-1)^{h+i+j} \delta^{-\theta(2+i)}\left(\begin{array}{c}
\theta-1 \\
h
\end{array}\right)\left(\begin{array}{c}
i+1 \\
i
\end{array}\right)\left(\begin{array}{c}
\theta i \\
j
\end{array}\right) e^{-\eta(h+j+1) x} .
\end{aligned}
$$

Therefore substituting (26) and (27) into (24) gives the density of the ith order statistics of the ALLoGExp distribution as

$$
\begin{aligned}
f_{X_{(i)}}(x) & =\frac{n !\left[\delta^{\theta}+1\right]^{i+\ell} \delta^{\theta} \theta \eta}{(i-1) !(n-i) !} \sum_{h=0}^{\theta-1} \sum_{i=0}^{2} \sum_{j=0}^{\theta i} \sum_{k=0}^{\theta(i+\ell-1)} \sum_{\ell=0}^{n-i} \sum_{m=0}^{i+\ell-1} \sum_{n=0}^{\theta m}(-1)^{k+\ell+m+n} \\
& \times \delta^{-\theta(2 i+\ell+m+1)}\left(\begin{array}{c}
\theta-1 \\
h
\end{array}\right)\left(\begin{array}{c}
i+1 \\
i
\end{array}\right)\left(\begin{array}{c}
\theta i \\
j
\end{array}\right)\left(\begin{array}{c}
\theta(i+\ell-1) \\
k
\end{array}\right)\left(\begin{array}{c}
n-i \\
\ell
\end{array}\right) \\
& \times\left(\begin{array}{c}
i+\ell+m-2 \\
m
\end{array}\right)\left(\begin{array}{c}
\theta m \\
n
\end{array}\right) e^{-\eta(h+j+k+n+1) x} .
\end{aligned}
$$

The density of the smallest order statistics of the ALLoGExp distribution is given by;

$$
\begin{aligned}
f_{X_{(1)}}(x) & =n\left[\delta^{\theta}+1\right]^{1+\ell} \delta^{\theta} \theta \eta \sum_{h=0}^{\theta-1} \sum_{j=0}^{\theta} \sum_{k=0}^{\theta \ell} \sum_{\ell=0}^{n-1} \sum_{m=0}^{\ell} \sum_{n=0}^{\theta m}(-1)^{k+\ell+m+n} \delta^{-\theta(3+\ell+m)} \\
& \times\left(\begin{array}{c}
\theta-1 \\
h
\end{array}\right)\left(\begin{array}{c}
\theta \\
j
\end{array}\right)\left(\begin{array}{c}
\theta \ell \\
k
\end{array}\right)\left(\begin{array}{c}
n-1 \\
\ell
\end{array}\right)\left(\begin{array}{c}
\ell+m-1 \\
m
\end{array}\right)\left(\begin{array}{c}
\theta m \\
n
\end{array}\right) e^{-\eta(h+j+k+n+1) x},
\end{aligned}
$$

while the density of largest order statistics of the ALLoGExp distribution is given by;

$$
\begin{aligned}
f_{X_{(n)}}(x) & =n\left[\delta^{\theta}+1\right]^{n+\ell} \delta^{\theta} \theta \eta \sum_{h=0}^{\theta-1} \sum_{j=0}^{\theta n} \sum_{k=0}^{\theta(n+\ell-1)} \sum_{m=0}^{n+\ell-1} \sum_{n=0}^{\theta m}(-1)^{k+\ell+m+n} \delta^{-\theta(\ell+m+2 n+1)}\left(\begin{array}{c}
\theta-1 \\
h
\end{array}\right) \\
& \times\left(\begin{array}{c}
n+1 \\
n
\end{array}\right)\left(\begin{array}{c}
\theta n \\
j
\end{array}\right)\left(\begin{array}{c}
\theta(n+\ell-1) \\
k
\end{array}\right)\left(\begin{array}{c}
\ell+m+n-2 \\
m
\end{array}\right)\left(\begin{array}{c}
\theta m \\
n
\end{array}\right) e^{-\eta(h+j+k+n+1) x} .
\end{aligned}
$$




\subsubsection{Moment of the Order Statistics}

Theorem 2.4. If $X$ follows the ALLoGExp distribution with pdf of the ith order statistics $f_{X_{(i)}}(x)$ then, its pth crude moment is given by;

$$
E\left(X_{i, n}^{p}\right)=\frac{\digamma_{x_{(i)}} \Gamma(p+1)}{[\eta(h+j+k+n+1)]^{p+1}},
$$

where $\Gamma(\cdot)$ is the gamma function and $\digamma_{x_{(i)}}$ is defined below.

Proof. By the definition of moment of a continuous random variable we have,

$$
E\left(X_{i, n}^{p}\right)=\digamma_{x_{(i)}} \int_{0}^{\infty} x^{p} e^{-\eta(h+j+k+n+1) x} d x
$$

by substituting $y=\eta(h+j+k+n+1) x$ in (28) we have

$$
\begin{aligned}
E\left(X_{i, n}^{p}\right) & =\frac{\digamma_{x_{(i)}}}{[\eta(h+j+k+n+1)]^{p+1}} \int_{0}^{\infty} y^{p} e^{-y} d y \\
& =\frac{\digamma_{x_{(i)}} \Gamma(p+1)}{[\eta(h+j+k+n+1)]^{p+1}} .
\end{aligned}
$$

Where,

$$
\begin{aligned}
\digamma_{x_{(i)}} & =\frac{n !\left[\delta^{\theta}+1\right]^{i+\ell} \delta^{\theta} \theta \eta}{(i-1) !(n-i) !} \sum_{h=0}^{\theta-1} \sum_{i=0}^{2} \sum_{j=0}^{\theta i} \sum_{k=0}^{\theta(i+\ell-1)} \sum_{\ell=0}^{n-i} \sum_{m=0}^{i+\ell-1} \sum_{n=0}^{\theta m}(-1)^{k+\ell+m+n} \\
& \times \delta^{-\theta(2 i+\ell+m+1)}\left(\begin{array}{c}
\theta-1 \\
h
\end{array}\right)\left(\begin{array}{c}
i+1 \\
i
\end{array}\right)\left(\begin{array}{c}
\theta i \\
j
\end{array}\right)\left(\begin{array}{c}
\theta(i+\ell-1) \\
k
\end{array}\right)\left(\begin{array}{c}
n-i \\
\ell
\end{array}\right) \\
& \times\left(\begin{array}{c}
i+\ell+m-2 \\
m
\end{array}\right)\left(\begin{array}{c}
\theta m \\
n
\end{array}\right) .
\end{aligned}
$$

\section{Estimation}

Here, we estimate the parameters of the ALLoGExp distribution by the method of maximum likelihood estimation. Suppose the random sample $x_{1}, x_{2}, x_{3}, \ldots, x_{n}$ of size $n$ is drawn from the ALLoGExp distribution with $p d f f(x)$ in (7) then the maximum likelihood estimation (mle) procedure for estimating its parameters is as follows:

The likelihood $(\mathcal{L})$ equation is given by;

$$
\begin{aligned}
\mathcal{L} & =\prod_{i=1}^{n}\left[\delta^{\theta}+1\right] \delta^{\theta} \theta \eta e^{-\eta x_{i}}\left(1-e^{-\eta x_{i}}\right)^{\theta-1}\left[\delta^{\theta}+\left(1-e^{-\eta x_{i}}\right)^{\theta}\right]^{-2} \\
& =\left[\delta^{\theta}+1\right]^{n}\left(\delta^{\theta} \theta \eta\right)^{n} e^{-\eta \sum_{i=1}^{n} x_{i}} \prod_{i=1}^{n}\left(1-e^{-\eta x_{i}}\right)^{\theta-1}\left[\delta^{\theta}+\left(1-e^{-\eta x_{i}}\right)^{\theta}\right]^{-2}
\end{aligned}
$$

and the log-likelihood function is given by;

$$
\begin{aligned}
\mathscr{L}= & n \log \left[\delta^{\theta}+1\right]+n \log \left(\delta^{\theta} \theta \eta\right)-\eta \sum_{i=1}^{n} x_{i}+(\theta-1) \sum_{i=1}^{n} \log \left(1-e^{-\eta x_{i}}\right) \\
& -2 \sum_{i=1}^{n} \log \left[\delta^{\theta}+\left(1-e^{-\eta x_{i}}\right)^{\theta}\right] .
\end{aligned}
$$

Taking the partial derivatives of (30) with respect to $\delta, \theta$ and $\eta$ gives

$$
\frac{\partial \mathscr{L}}{\partial \delta}=\frac{n \theta \delta^{\theta-1}}{\delta^{\theta}+1}+\frac{n \theta}{\delta}-2 \sum_{i=1}^{n} \frac{\theta \delta^{\theta-1}}{\delta^{\theta}+\left(1-e^{-\eta x_{i}}\right)^{\theta}},
$$




$$
\begin{aligned}
\frac{\partial \mathscr{L}}{\partial \theta} & =\frac{n \delta^{\theta} \log (\delta)}{\delta^{\theta}+1}+n \log (\delta)+\frac{n}{\theta}+\sum_{i=1}^{n} \log \left(1-e^{-\eta x_{i}}\right) \\
& -2 \sum_{i=1}^{n} \frac{\delta^{\theta} \log (\delta)+\left(1-e^{-\eta x_{i}}\right)^{\theta} \log \left(1-e^{-\eta x_{i}}\right)}{\delta^{\theta}+\left(1-e^{-\eta x_{i}}\right)^{\theta}}
\end{aligned}
$$

and

$$
\frac{\partial \mathscr{L}}{\partial \eta}=\frac{n}{\eta}-\sum_{i=1}^{n} x_{i}+(\theta-1) \sum_{i=1}^{n} \frac{x_{i} e^{-\eta x_{i}}}{1-e^{-\eta x_{i}}}-2 \sum_{i=1}^{n} \frac{\theta x_{i} e^{-\eta x_{i}}\left(1-e^{-\eta x_{i}}\right)^{\theta-1}}{\delta^{\theta}+\left(1-e^{-\eta x_{i}}\right)^{\theta}} .
$$

Furthermore, setting (31)-(33) to zero results to a system of three equations in three unknowns which has no analytical solutions.

However, the estimates $\hat{\delta}, \hat{\theta}$ and $\hat{\eta}$ can only be obtained by solving (31)-(33) by some non linear numerical optimization methods eg.; the Newton-Raphson or quasi-Newton-Raphson's technique.

\section{Reliability}

The reliability function $R(x)$ is an important tool in reliability analysis for characterizing life phenomena. The reliability function is mathematically expressed as $1-F(x)$. Under certain predefined conditions $R(x)$ generally gives the estimate of the probability that, a system will not fail given that it has operated without failure up to time $x$. The reliability function of the ALLoGExp distribution is given by;

$$
R(x)=1-\frac{\left(\delta^{\theta}+1\right)\left(1-e^{-\eta x}\right)^{\theta}}{\delta^{\theta}+\left(1-e^{-\eta x}\right)^{\theta}} ; x \geq 0, \delta, \theta, \eta>0 .
$$

Another important reliability characteristics is the failure rate function $h(x)$. The failure rate function gives the probability of failure, for a system that has not failed up-to time $x$. The failure rate function is mathematically expressed as $f(x) / R(x)$. The failure rate function of the ALLoGExp distribution is given by;

$$
h(x)=\frac{\left(\delta^{\theta}+1\right) \delta^{\theta} \theta \eta e^{-\eta x}\left(1-e^{-\eta x}\right)^{\theta-1}}{\left[\delta^{\theta}+\left(1-e^{-\eta x}\right)^{\theta}\right]\left[\delta^{\theta}+\left(1-e^{-\eta x}\right)^{\theta}-\left(\delta^{\theta}+1\right)\left[1-e^{-\eta x}\right]^{\theta}\right]} ; x \geq 0, \delta, \theta, \eta>0 .
$$

\subsection{Shapes and Asymptotics}

(a) The $p d f$ of the ALLoGExp distribution could either be a unimodal or monotonic decreasing function of $x$ depending on the value of $\delta$ and $\theta$, while $F(x)$ is an increasing function of $x$ for all possible values of $\delta$ and $\theta$ parameters (see; Figures 1 and 2), and the asymptotic behaviour of the $p d f$ is

$$
\lim _{x \rightarrow 0} f(x)= \begin{cases}0, & \text { if } \theta>1, \\ \eta(\delta+1) / \delta, & \text { if } \theta=1, \\ \infty, & \text { if } \theta<1,\end{cases}
$$

and the asymptotic behaviour of the $c d f$ is $\lim _{x \rightarrow \infty} F(x)=1$ while $\lim _{x \rightarrow 0} F(x)=0$.

(b) The reliability function $R(x)$ of the $A L L o G E x p$ distribution is generally a monotonic decreasing function of $x$ for all possible values of $\delta$ and $\theta$ parameters (see; Figure 3), and $\lim _{x \rightarrow \infty} R(x)=0$ while $\lim _{x \rightarrow 0} R(x)=1$.

(c) The failure rate function $(f r f) h(x)$ of the ALLoGExp distribution could be a decreasing, increasing or upside-down bathtub shaped function of $x$ depending on the value of the $\delta$ and $\theta$ parameters (see; Figure 4), and $\lim _{x \rightarrow \infty} h(x)=$ 0 , while

$$
\lim _{x \rightarrow 0} h(x)= \begin{cases}0, & \text { if } \theta>1, \\ \eta(\delta+1) / \delta, & \text { if } \theta=1 \\ \infty, & \text { if } \theta<1 .\end{cases}
$$



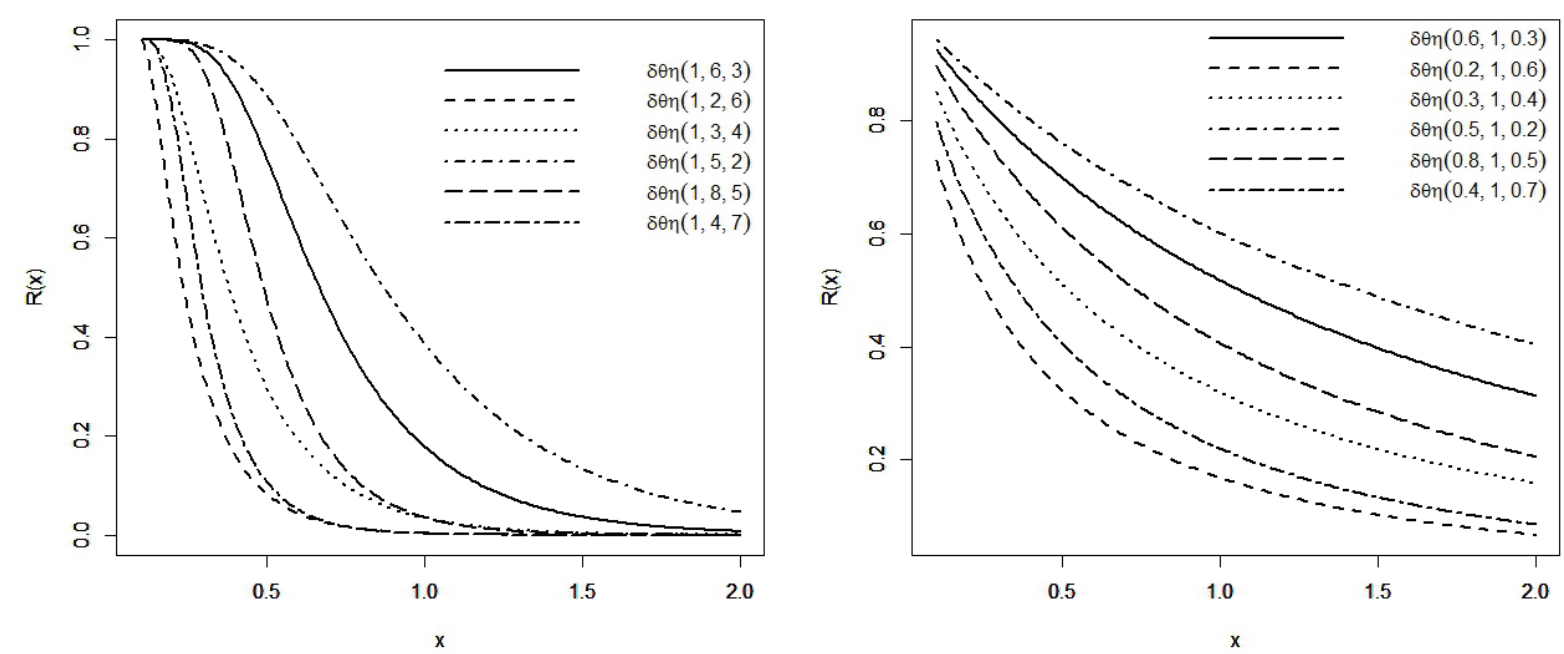

Figure 3. Possible shapes of the reliability function $R(x)$ for some parameter values.
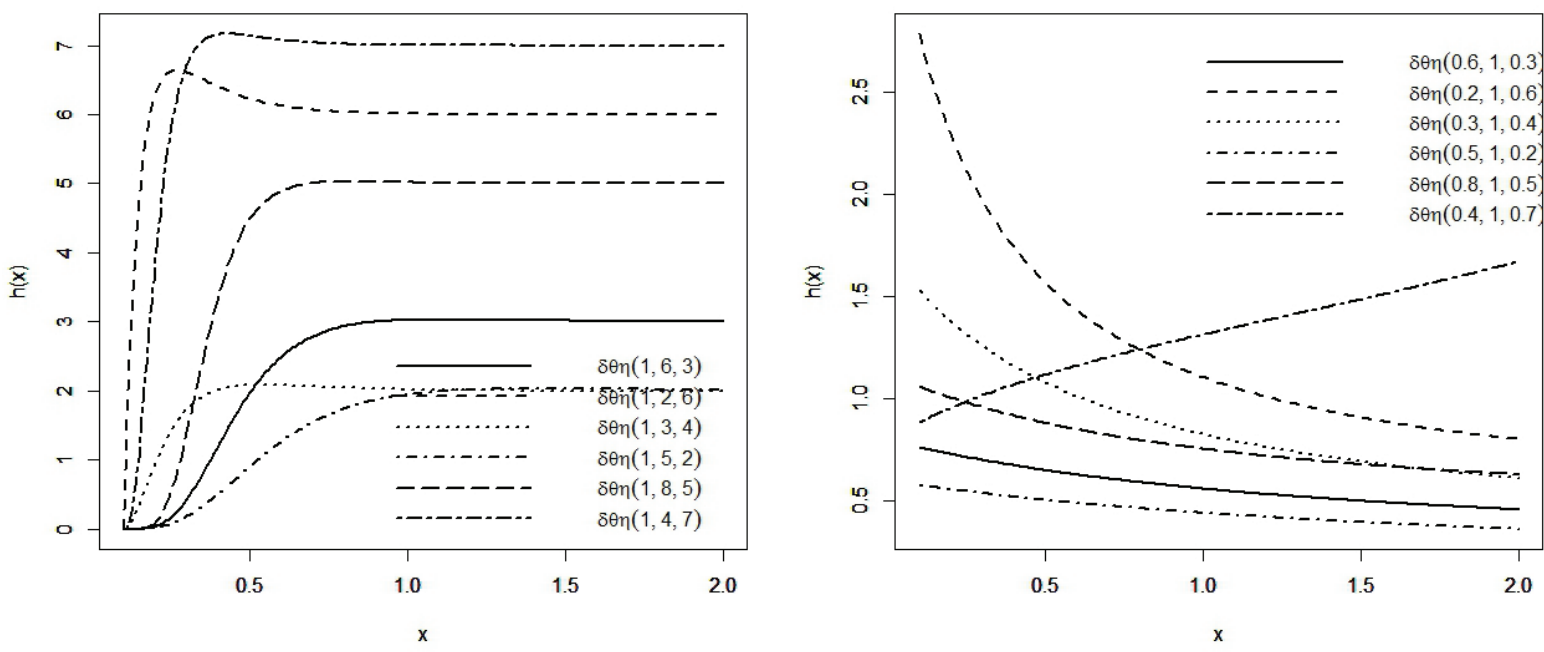

Figure 4. Possible shapes of the hazard rate function $h(x)$ for some parameter values.

The loss of memory property of the exponential distribution and the shape limitation of its failure rate are well known. The major advantage of the new distribution over the baseline model is its added tail and skewness flexibility due to the presence of $\delta$ and $\theta$. The ALLoGExp is suitable for modelling lifetime data sets with increasing, decreasing, unimodal and upside-down bathtub failure rate characteristics.

\section{Monte-Carlo Simulation}

In this section, we investigate the consistency of the mle estimates of the ALLoGExp distribution with different sample size $(n)$, through a Monte-Carlo study. The simulation procedure as outlined below was implemented in $R$ (Statistical software):

1. simulate a random sample of size $n$ from the ALLoGExp distribution with parameters $\delta=0.5, \theta=4.0$ and $\eta=6.0$ using the inversion of the $c d f$ method with Equation (8).

2. compute the mle of the parameters of the ALLoGExp distribution. 
3. repeat steps 1-2 5000 times.

4. compute the mean, standard deviation (standard error), bias and mean square error ( $m s e$ ) of the 5000 estimates of each of the parameters $(\delta, \theta$ and $\eta)$.

5. repeat steps $1-4$ for different sample sizes ( $n=150,250, \ldots, 950$.$) .$

Results from the Monte-Carlo simulation study are tabulated in Tables 1 and 2.

Table 1. Simulation results of the estimates and standard errors of the ALLoGExp distribution parameters for different sample sizes

\begin{tabular}{c|cccccc}
\hline $\boldsymbol{n}$ & $\hat{\boldsymbol{\delta}}$ & $\hat{\boldsymbol{\theta}}$ & $\hat{\boldsymbol{\eta}}$ & $s e_{\hat{\boldsymbol{\delta}}}$ & $s e_{\hat{\boldsymbol{\theta}}}$ & $s e_{\hat{\boldsymbol{\eta}}}$ \\
\hline 150 & 0.610892 & 4.067068 & 6.751090 & 1.658690 & 0.598297 & 3.510499 \\
250 & 0.529417 & 4.054277 & 6.540886 & 0.305542 & 0.464951 & 2.948877 \\
350 & 0.515020 & 4.031474 & 6.404858 & 0.169692 & 0.406658 & 2.484260 \\
450 & 0.501594 & 4.003761 & 6.180374 & 0.159987 & 0.372123 & 2.285624 \\
550 & 0.511001 & 4.027797 & 6.299262 & 0.139514 & 0.332690 & 2.060127 \\
650 & 0.498788 & 3.997763 & 6.094940 & 0.131292 & 0.294331 & 1.894563 \\
750 & 0.499263 & 3.996266 & 6.089056 & 0.119744 & 0.281376 & 1.757239 \\
850 & 0.506678 & 4.013708 & 6.171866 & 0.108199 & 0.253175 & 1.602942 \\
950 & 0.507347 & 4.011057 & 6.186832 & 0.108731 & 0.257343 & 1.612633 \\
\hline
\end{tabular}

Table 2. Simulation results of the bias and mse of the ALLoGExp distribution parameters for different sample sizes

\begin{tabular}{c|cccccc}
\hline $\boldsymbol{n}$ & bias $_{\hat{\boldsymbol{\delta}}}$ & bias $_{\hat{\boldsymbol{\theta}}}$ & bias $_{\hat{\boldsymbol{\eta}}}$ & mse $_{\hat{\boldsymbol{\delta}}}$ & mse $_{\hat{\boldsymbol{\theta}}}$ & sse $_{\hat{\boldsymbol{\theta}}}$ \\
\hline 150 & 0.110892 & 0.067068 & 0.751090 & 2.760798 & 0.362099 & 12.875415 \\
250 & 0.029417 & 0.054277 & 0.540886 & 0.094128 & 0.218909 & 8.979740 \\
350 & 0.015020 & 0.031474 & 0.404858 & 0.028992 & 0.166196 & 6.329287 \\
450 & 0.001594 & 0.003761 & 0.180374 & 0.025573 & 0.138351 & 5.251386 \\
550 & 0.011001 & 0.027797 & 0.299262 & 0.019566 & 0.111345 & 4.329437 \\
650 & -0.001212 & -0.002237 & 0.094940 & 0.017222 & 0.086549 & 3.594793 \\
750 & -0.000738 & -0.003734 & 0.089056 & 0.014325 & 0.079107 & 3.092733 \\
850 & 0.006678 & 0.013708 & 0.171866 & 0.011740 & 0.064221 & 2.596392 \\
950 & 0.007347 & 0.011057 & 0.186832 & 0.011865 & 0.066282 & 2.632891 \\
\hline
\end{tabular}

The simulation results in Table 1 indicates that the mle estimates of the ALLoGExp distribution is generally consistent for $n$; while the standard error, bias and $m s e$ approaches zero as $n$ becomes large.

\section{Application}

This section illustrates the applicability and flexibility of the ALLoGExp distribution with a real data set. The goodness of fit of the new lifetime distribution would be assessed by a comparison of its performance in modelling real data with the following five distributions:

(i) The exponentiated exponential (EE) distribution due to Gupta and Kundu (1999),

$$
f(x)=\alpha \eta\left(1-e^{-\eta x}\right)^{\alpha-1} e^{-\eta x} ; \quad x, \alpha, \eta>0 .
$$

(ii) The Log-logistic (LLo) distribution,

$$
f(x)=\frac{\frac{\theta}{\delta}\left(\frac{x}{\delta}\right)^{\theta-1}}{\left[1+\left(\frac{x}{\delta}\right)^{\theta}\right]^{2}} ; \quad x, \theta, \delta>0 .
$$

(iii) The Lindley exponential (LE) distribution due to Bhati et al. (2006),

$$
f(x)=\frac{\phi^{2} \eta e^{-\eta x}\left(1-e^{-\eta x}\right)^{\phi-1}\left(1-\log \left(1-e^{-\eta x}\right)\right)}{1+\phi} ; x, \phi, \eta>0 .
$$


(iv) The Nadarajah-Haghighi exponential (NHE) distribution due to Nadarajah and Haghighi (2015),

$$
f(x)=\beta \eta(1+\beta x)^{\eta-1} e^{1-(1+\beta x)^{\eta}} ; \quad x, \beta, \eta>0 .
$$

(v) The standard one parameter exponential (Exp) distribution,

$$
f(x)=\eta e^{-\eta x} ; \quad x, \eta>0 .
$$

Comparison of the models would be based on the following information criteria statistics:

- the Akaike information criterion (AIC) statistic, Akaike (1992),

$$
A I C=-2 \hat{\mathscr{L}}+2 k
$$

- the AIC with a correction statistic (AICc), Sugiura (1978),

$$
A I C c=A I C+\frac{2 k(k+1)}{n-k-1}
$$

- and the Hannan-Quinn information criterion (HQC) statistic, Hannan and Quinn (1979),

$$
H Q C=-2 \hat{\mathscr{L}}+2 k \log \log (n)
$$

Where $-\hat{\mathscr{L}}, k$, and $n$ corresponds to the estimate of the model minimized log-likelihood function, number of model parameters and sample size, respectively.

We have also considered some goodness-of-fit test based on the empirical distribution function; namely, the KolmogorovSmirnov (K-S) statistics by Henze and Meintanis (2005) and Liao-Shimokawa statistics due to Liao and Shimokawa (1999). They are given by;

$$
K-S=\max \left(\frac{i}{n}-F\left(x_{(i)}\right), F\left(x_{(i)}\right)-\frac{i-1}{n}\right),
$$

and

$$
L-S=\frac{1}{\sqrt{n}} \sum_{i=1}^{n} \frac{\max \left(\frac{i}{n}-F\left(x_{(i)}\right), F\left(x_{(i)}\right)-\frac{i-1}{n}\right)}{\sqrt{F\left(x_{(i)}\right)\left(1-F\left(x_{(i)}\right)\right)}},
$$

respectively. Where $n$ is the number of observations in the data set, $1 \leq i \leq n$ and $F\left(x_{(i)}\right)$ is the $c d f$ of the fitted distribution under the ascending ordered data.

The real and uncensored data set in Table 3 is on the active repair times in hours for an airborne communication transceiver. The data set was originally reported by Von Alven (1964) and later Chhikara and Folks (1977) used it on the inverse Gaussian distribution.

Table 3. Active repair time data

\begin{tabular}{ccccccccccccccccc}
\hline 0.2 & 0.3 & 0.5 & 0.5 & 0.5 & 0.5 & 0.6 & 0.6 & 0.7 & 0.7 & 0.7 & 0.8 & 0.8 & 1.0 & 1.0 & 1.0 & 1.0 \\
1.1 & 1.3 & 1.5 & 1.5 & 1.5 & 1.5 & 2.0 & 2.0 & 2.2 & 2.5 & 2.7 & 3.0 & 3.0 & 3.3 & 3.3 & 4.0 & 4.0 \\
4.5 & 4.7 & 5.0 & 5.4 & 5.4 & 7.0 & 7.5 & 8.8 & 9.0 & 10.3 & 22.0 & 24.5 & & & & & \\
\hline
\end{tabular}

The results of the fits are listed in Table 4. The plots in Figure 5 shows the $p d f$ and $c d f$ of the fitted theoretical distributions superimposed on the empirical density and distribution function, respectively. 
Table 4. Results

\begin{tabular}{c|ccccccc}
\hline Models & Estimates & $-\hat{\mathscr{L}}$ & AIC & AICc & HQC & K-S & L-S \\
\hline ALLoGExp & & & & & & & \\
$\hat{\delta}$ & 0.1856 & & & & & & \\
$\hat{\theta}$ & 1.5666 & 100.7382 & 207.4763 & 208.0477 & 209.5314 & 0.01157303 & 0.01701021 \\
$\hat{\eta}$ & 0.0995 & & & & & & \\
$\mathbf{L E}$ & & & & & & & \\
$\hat{\phi}$ & 1.2803 & 103.7034 & 211.4067 & 211.6858 & 212.7768 & 0.01973152 & 0.06499466 \\
$\hat{\eta}$ & 0.2401 & & & & & & \\
$\mathbf{E E}$ & & & & & & & \\
$\hat{\alpha}$ & 0.9583 & 104.9829 & 213.9658 & 214.2449 & 215.3359 & 0.02043486 & 0.08348181 \\
$\hat{\eta}$ & 0.2694 & & & & & & \\
$\mathbf{N H E}$ & & & & & & & \\
$\hat{\beta}$ & 0.6364 & 103.2059 & 210.4118 & 210.6909 & 211.7819 & 0.01476631 & 0.02616426 \\
$\hat{\eta}$ & 0.6348 & & & & & & \\
$\mathbf{L L o}$ & & & & & & & \\
$\hat{\theta}$ & 0.6477 & 101.1710 & 206.3421 & 208.3421 & 205.4908 & 0.01847885 & 0.02023060 \\
$\hat{\delta}$ & 0.6257 & & & & & & \\
$\mathbf{E x p}$ & & & & & & & \\
$\hat{\eta}$ & 0.2773 & 105.0062 & 212.0124 & 212.1033 & 212.6974 & 0.0206178 & 0.09083219 \\
\hline
\end{tabular}
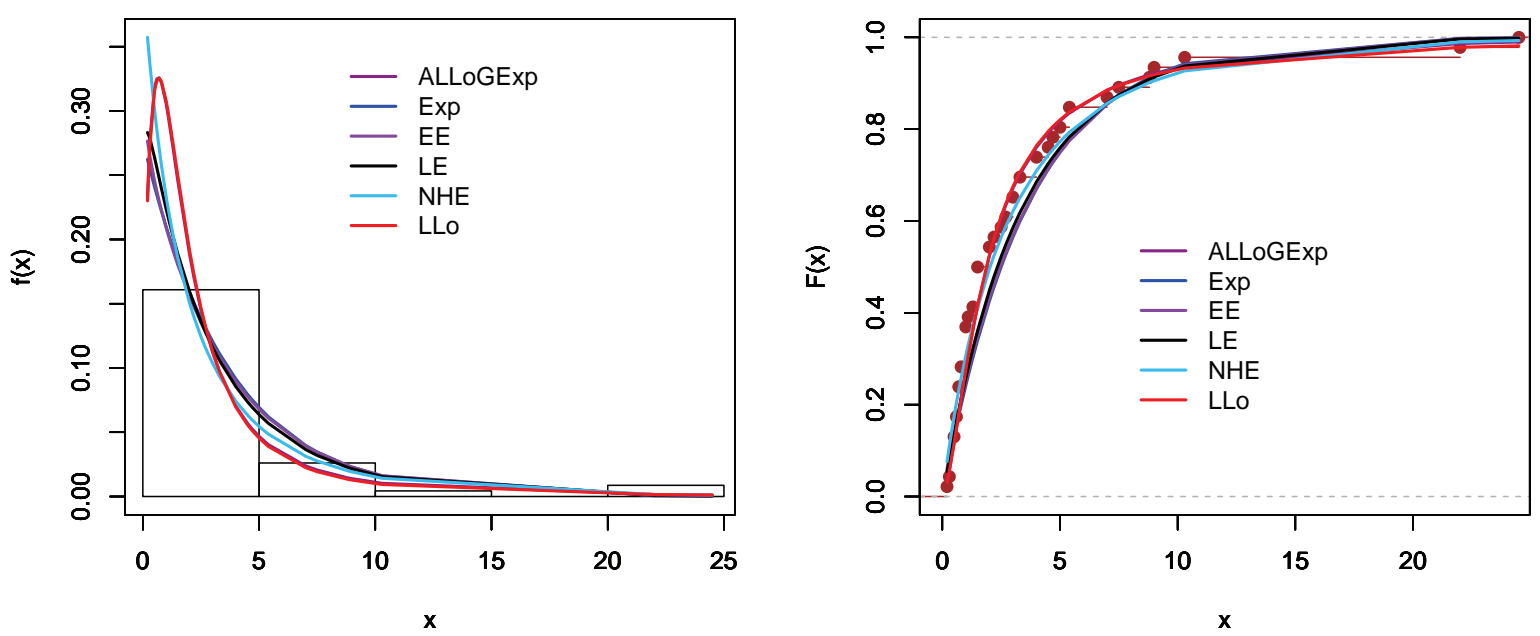

Figure 5. The estimated $p d f F(x)$ (left panel) and $c d f F(x)$ (right panel) plots of the fitted distributions superimposed on the empirical $p d f F(x)$ and $c d f F(x)$ of the active repair time data.

\section{Concluding Remarks}

In this paper we have introduced a new generator of distributions called the adjusted log-logistic generalized (ALLoG) distribution and a new lifetime distribution-the adjusted log-logistic generalized exponential (ALLoGExp) distribution is also introduced as a sub-model of the $A L L o G$ distribution. The new lifetime distribution generalizes the exponential (Exp) distribution. We have given explicit expressions of some of its basic statistical properties such as the probability density function, cumulative density function, $k$ th raw moment, mean, variance, coefficient of variation, skewness, kurtosis, moment generating function, pth quantile function, the ith order statistics, and the Rényi's entropy measure. Also, some of its reliability characteristics like the reliability function and the failure rate function were provided; the failure rate could be monotonically decreasing, increasing or upside-down bathtub shaped depending on the value of the scale parameter $\delta$ and shape parameter $\theta$. Estimation of the model parameters was approached through the method of maximum 
likelihood estimation mle and the stability of the mle estimates was verified through a Monte-Carlo simulation study. The applicability and goodness of fit of the ALLoGExp distribution was illustrated with the active repair times data and the results based on the AICc, K-S and L-S statistics shows that the ALLoGExp distribution provides a better fit than the Exp, $E E, L E, L L o$, and $N H$ distribution, also, the density plot of the ALLoGExp distribution comparatively provides the best fit to the histogram of the empirical data. We strongly recommend the ALLoGExp distribution for effective modelling of life time data because of its flexible failure rate characteristics.

\section{Competing Interest}

The authors declare no competition of interests.

\section{Acknowledgements}

The authors are thankful to the editor and two anonymous referees for their careful reading of the manuscript and invaluable comments and suggestions which significantly improved the content of this paper.

\section{References}

Alzaatreh, A., Lee, C., \& Famoye, F. (2013). A new method for generating families of continuous distributions. Metron, 7l(1), 63-79. https://doi.org/10.1007/s40300-013-0007-y

Akaike, H. (1992). Information Theory and an Extension of the Maximum Likelihood Principle. In Breakthroughs in Statistics (pp. 610-624). Springer New York. https://doi.org/10.1007/978-1-4612-0919-5-38

Bhati, D., Malik, M. A., \& Vaman, H. J. (2015). Lindley-Exponential distribution: properties and applications. METRON, 73(3), 335-357. https://doi.org/10.1007/s40300-015-0060-9

Chhikara, R. S., \& Folks, J. L. (1977). The inverse Gaussian distribution as a lifetime model. Technometrics, 19(4), 461-468. https://doi.org/10.1080/00401706.1977.10489586

Cordeiro, G. M., \& de Castro, M. (2011). A new family of generalized distributions. Journal of statistical computation and simulation, 81(7), 883-898. https://doi.org/10.1080/00949650903530745

Eugene, N., Lee, C., \& Famoye, F. (2002). Beta-normal distribution and its applications. Communications in StatisticsTheory and methods, 31(4), 497-512. https://doi.org/10.1081/STA-120003130

Gupta, R. D., \& Kundu, D. (1999). Theory \& Methods: Generalized Exponential Distributions. Australian $\mathcal{E}$ New Zealand Journal of Statistics, 41(2), 173-188. https://doi.org/10.1111/1467-842X.00072

Hannan, E. J., \& Quinn, B. G. (1979). The determination of the order of an autoregression. Journal of the Royal Statistical Society. Series B (Methodological), 190-195.

Henze, N., \& Meintanis, S. G. (2005). Recent and classical tests for exponentiality: a partial review with comparisons. Metrika, 61(1), 29-45. https://doi.org/10.1007/s001840400322

Liao, M., \& Shimokawa, T. (1999). A new goodness-of-fit test for type-I extreme-value and 2-parameter Weibull distributions with estimated parameters. Optimization, 64(1), 23-48.

Nadarajah, S., \& Kotz, S. (2006). The beta exponential distribution. Reliability engineering $\mathcal{E}$ system safety, 91(6), 689-697. https://doi.org/10.1016/j.ress.2005.05.008

Nadarajah, S., \& Rocha, R. (2016). Newdistns: An R Package for New Families of Distributions. Journal of Statistical Software, 69(i10). https://doi.org/10.18637/jss.v069.i10

Ristić, M. M., \& Balakrishnan, N. (2012). The gamma-exponentiated exponential distribution. Journal of Statistical Computation and Simulation, 82(8), 1191-1206. https://doi.org/10.1080/00949655.2011.574633

Sugiura, N. (1978). Further analysts of the data by akaike's information criterion and the finite corrections: Further analysts of the data by akaike's. Communications in Statistics-Theory and Methods, 7(1), 13-26.

Von Alven, W. H. (Ed.). (1964). Reliability engineering. Prentice Hall.

Zografos, K., \& Balakrishnan, N. (2009). On families of beta-and generalized gamma-generated distributions and associated inference. Statistical Methodology, 6(4), 344-362. https://doi.org/10.1016/j.stamet.2008.12.003

\section{Copyrights}

Copyright for this article is retained by the author(s), with first publication rights granted to the journal.

This is an open-access article distributed under the terms and conditions of the Creative Commons Attribution license (http://creativecommons.org/licenses/by/4.0/). 